\title{
Exploring Key Technologies of Multi-Sensor Data Fusion
}

\author{
Li Wang ${ }^{1,}$, , Hongxia Guo ${ }^{2}$ \\ ${ }^{1}$ Department of Energy Engineering, Yulin University, Yulin 719000, Shannxi, China \\ a278116312@qq.com
}

\begin{abstract}
Keywords: multiple sensors; data fusion; key technology
Abstract. In the mid and late 20th century, with the rapid development of computer technology, control technology, electronic technology and sensor technology, etc., multi-sensor data fusion technology emerged. Data acquired by multi-sensor data fusion technology has multiple levels and grades, which is of more significance for practical application. Centered on multi-sensor data fusion technology, this paper first introduces data fusion, and then elaborates on key technologies of multi-sensor data fusion, in the hope of promoting further development of these technologies.
\end{abstract}

\section{Introduction}

Compared with a single sensor, the application of multi-sensor data fusion technology in target identification, detection, tracking and so on can effectively enhance the reliability and validity of data, ensure data accuracy and promote the robustness and real time of system operation and information use. At present, this technology has been widely used in military, civil and other fields, such as robot, marine monitoring, medical diagnosis, transport and image processing and so on. Thus, to study multi-sensor data fusion technology is of important practical significance for the improvement of technical content and promotion of rapid development of various industries.

\section{The Definition, Structures and Fusion Levels of Data Fusion}

\subsection{The definition of data fusion}

Multi-sensor data fusion technology is derived from the basic function of human and animal information integration systems, namely, people integrate information from their eyes, ears, nerve and other sensors naturally and detect, estimate and understand objects, environment and mechanism of ongoing events around them, by thinking with brain and perceiving with body. By synthesizing multiple sensors and making full use of them, they can acquire diverse, multi-grade and multi-level information comprehensively, describe and explain the measured object in a uniform way [1]. However, there is no unified definition about the concept of data fusion. A representative definition is: a series of processes used to detect, estimate, correlate and associate data from multiple sources using multiple sensors. It is a multi-level, multi-source and multi-grade data process. The structures and levels of fusion are shown in Tab. 1.

Tab. 1 Descriptions about Structures and Levels of Multi-Sensor Data Fusion

\begin{tabular}{|c|c|c|c|c|}
\hline Data Fusic & & Description & & \\
\hline Data & Fusion & Serial structure & Parallel structure & Network structure \\
\hline Structure & & $\begin{array}{l}\text { Centralized } \\
\text { structure }\end{array}$ & $\begin{array}{l}\text { Distributed } \\
\text { structure }\end{array}$ & Hybrid structure \\
\hline $\begin{array}{l}\text { Data } \\
\text { Level }\end{array}$ & Fusion & Data-level fusion & $\begin{array}{l}\text { Feature-level } \\
\text { fusion }\end{array}$ & Decision-level fusion \\
\hline
\end{tabular}

\subsection{Data fusion structure}

To reduce information loss in a single sensor and improve the intelligent detection of system performance, operational reliability and information use is an advantage and characteristic of multi-sensor data fusion technology. From the relationship and fusion process between sensors and core information flow in data fusion, a data fusion structure is mainly composed of four parts, serial, 
parallel, serial-parallel and network. Serial and parallel structures are as shown in Figs. 1 and 2 respectively [2].

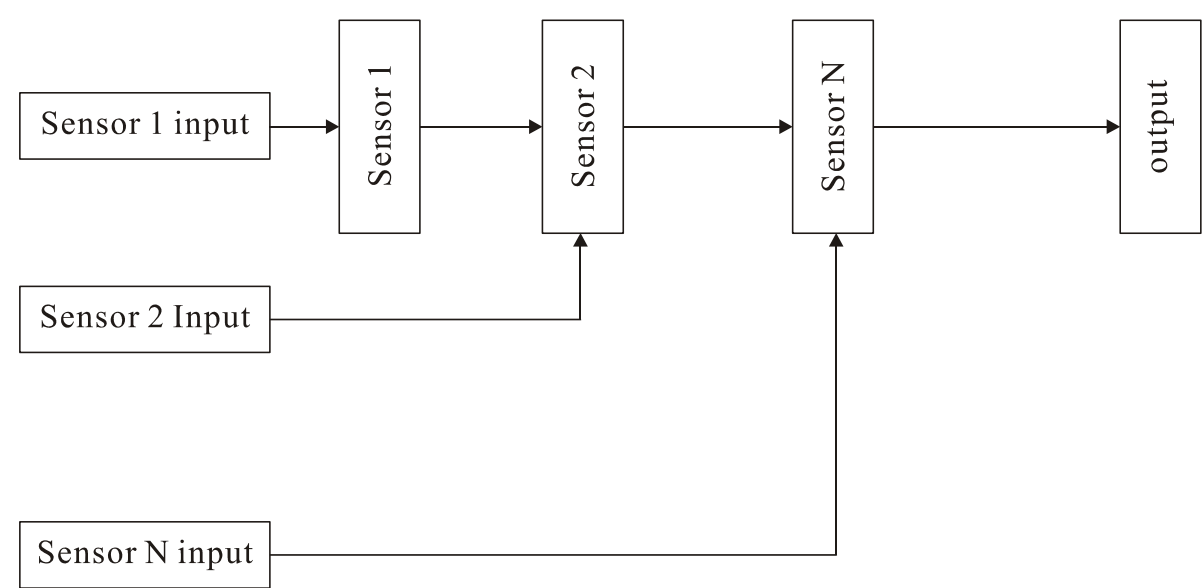

Fig.1 The Serial Structure of Multi-sensor Data Fusion

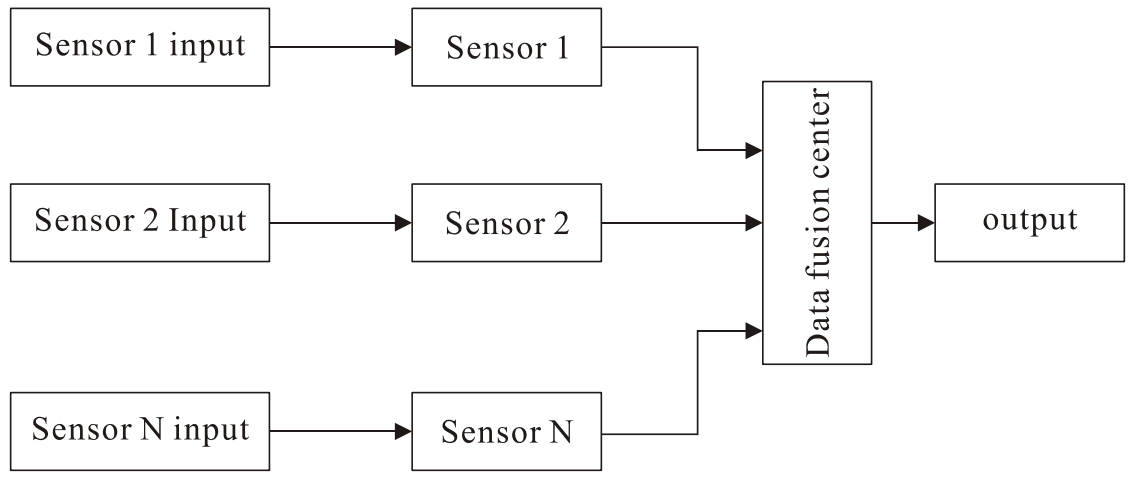

Fig. 2 The Parallel Structure of Multi-sensor Data Fusion

The serial structure of multi-sensor data fusion refers to fusing data from two sensors and then fusing the output with data acquired from another sensor. The fusion proceeds in the same manner until all sensor data are fused. Under this structure, all sensors in multi-sensor data fusion not only acquire data from a single sensor, but also fuse data. Besides, data output from the previous sensor has a significant impact on data output in the next sensor. The final output that fuses all previous sensors will be fused as the final conclusion of system [3]. The parallel structure of multi-sensor data fusion means that the fusion of all sensor output to the center is simultaneous and sensors are mutually independent. When all data are fused to the processing center, the center will process the fusion results as a whole, according to various data types using appropriate methods. Finally, results will be output.

On the other hand, data fusion also has common processing structures, i.e., centralized structure, distributed structure and hybrid structure.

2.3 Data fusion level

Multi-sensor data fusion is a multi-level data process. It has diverse and complex structures and applies to different information levels. In abstract terms, fusion levels are generally divided into three parts, data-level fusion, feature-level fusion and decision-level fusion, from low to high. Data-level fusion is at the lowest level of the whole hierarchy of multi-sensor data fusion, which is mainly used to process homogeneous data, fuse information acquired by sensors directly, extract and decide on features of fusion results. It has high precision and small information loss [4]. Feature-level fusion first extracts a set of feature data from original data acquired by various sensors and then fuses all sets of feature data. Feature-level fusion mainly describes the performance, function and behavior, etc. of the measured object. It is composed of target state information (Kalman filtering, multi hypothesis, etc.) and target feature information (parametric template, artificial neural network, etc.) Decision-level fusion is the highest level of multi-sensor data fusion. First of all, it preprocesses all types of data acquired by various sensors, makes an independent decision on the measured object. 
After that, it fuses all decision results. Finally, it obtains decision results with overall consistency. The advantages and disadvantages of the three levels are shown in Tab. 2.

Tab. 2 The Advantages and Disadvantages of Three Data Fusion Levels

\begin{tabular}{|c|c|c|}
\hline Data Fusion Level & Advantage & Disadvantage \\
\hline Data-level fusion & $\begin{array}{l}\text { High precision, small } \\
\text { information loss }\end{array}$ & $\begin{array}{l}\text { Poor anti-jamming ability, } \\
\text { poor real time }\end{array}$ \\
\hline $\begin{array}{l}\text { Decision-level } \\
\text { fusion }\end{array}$ & $\begin{array}{l}\text { Strong anti-jamming ability, } \\
\text { small traffic and low cost }\end{array}$ & $\begin{array}{l}\text { Large information loss and low } \\
\text { precision }\end{array}$ \\
\hline $\begin{array}{l}\text { Feature-level } \\
\text { fusion }\end{array}$ & $\begin{array}{l}\text { Favorable for the acquisition } \\
\text { of target feature and state } \\
\text { information, improving the } \\
\text { target identification ability of } \\
\text { system }\end{array}$ & $\begin{array}{l}\text { High demand for information } \\
\text { credibility, no fixed model to } \\
\text { follow }\end{array}$ \\
\hline
\end{tabular}

\section{Key Technologies of Multi-Sensor Data Fusion}

Multi-sensor data fusion is an integrated information processing system. Due to diverse structures and multiple levels of data fusion, there are many algorithms and methods for fusion, such as feature-level fusion technology based on fuzzy integral, fusion technology based on fuzzy logic, information gain measurement technology based on target feature, sensor management technology based on particle swarm algorithm, data association analysis algorithm, multi-source information fusion technology, etc., as shown in Tabs. 3 and 4.

Tab. 3 Key Algorithms and Technologies for Various Levels of Multi-Sensor Data Fusion

\begin{tabular}{ll}
\hline Data Fusion Level & Fusion Algorithm and Technology \\
\hline Data-level & $\begin{array}{l}\text { Data association analysis, feature extraction and identification, } \\
\text { etc. }\end{array}$
\end{tabular}

Feature-level Artificial neural network algorithm, feature extraction clustering, interacting multiple model, Kalman filtering, joint probabilistic data association, etc.

Decision-level Expert system, D-S evidence theory, fuzzy reasoning theory, etc.

Tab. 4 Key Technologies in JDL Multi-Sensor Data Fusion Model

\begin{tabular}{ll}
\hline JDL Model & Key Technologies \\
\hline Level 0 & Signal preprocessing, feature extraction \\
Level 1 & $\begin{array}{l}\text { Data registration, data association, detection, identification and sorting } \\
\text { algorithm, state estimation and tracking algorithm }\end{array}$ \\
Levels 2 and 3 & Threat assessment algorithm, situation assessment algorithm \\
Level 4 & Sensor management, fusion performance evaluation
\end{tabular}

To take feature-level fusion algorithm based on fuzzy integral for example, suppose there is a set $\mathrm{X}$ and a fuzzy density measure function $\mathrm{g}$. Then the fuzzy density measure of the set can be expressed as follows:

$$
g_{\lambda}(X)=\sum_{i=1}^{n} g_{1}+\lambda \sum_{i_{1}=1}^{n-1} \sum_{i_{2}=i_{1}+1}^{n} g_{i_{1}} g_{i_{2}}+\cdots+\lambda^{n-1} g_{1} g_{2} \cdots g_{n}
$$

Where $g \lambda(X)$ is the importance measure of set $X$. The boundary condition is $g(X)=1[5]$. In order to make the fusion results more reliable and precise, we need to combine fuzzy density with fuzzy membership degree and then fuse. That is, to extract suspicious targets and target features, calculate the feature quantity of suspicious targets, transform the feature quantity into fuzzy density and membership degree and finally judge the fusion results according to fuzzy rules. 


\section{Conclusion}

To sum up, multi-sensor data fusion technology is a new technology with broad adaptability, strong data acquisition ability and processing ability. It can provide technical support and data information required by many fields, such as remote sensing, target identification, tracking and medical diagnosis. However, at the current stage, algorithms and structures of multi-sensor data fusion are still imperfect. There are some defects and limitations with them, which require people to intensify research and improve, to promote the level of multi-sensor data fusion technology.

\section{Acknowledgements}

This work was supported by the Research of Gas Detection System Based on ZigBee and LabVIEW; and Municipal Science and Technology Bureau of Yulin City(Item number : 2015CXY-22-03)

\section{References}

[1] Safizadeh M S, Latifi S K. Using multi-sensor data fusion for vibration fault diagnosis of rolling element bearings by accelerometer and load cell[J]. Information Fusion, 2014, 18(4):1-8.

[2] Fortino G, Galzarano S, Gravina R, et al. A framework for collaborative computing and multi-sensor data fusion in body sensor networks[J]. Information Fusion, 2014, 22:50 - 70.

[3] Cappello F, Ramasamy S, Sabatini R. Multi-sensor Data Fusion Techniques for RPAS Navigation and Guidance[J]. AIAC 16: Multinatioinal Aerospace Programs-Benefits and Challenges, 2015:1-18.

[4] Frikha A, Moalla H. Analytic hierarchy process for multi-sensor data fusion based on belief function theory[J]. European Journal of Operational Research, 2015, 241(1):133-147.

[5] Liu B, Zhang F, Qu X. A method for improving the pose accuracy of a robot manipulator based on multi-sensor combined measurement and data fusion.[J]. Sensors, 2015, 15(4):7933-7952. 